\title{
Spontaneous Emergence of Spatio-Temporal Order in Class 4 Automata
}

\author{
Jason A.C. Gallas ${ }^{a, b, *}$ Hans J. Herrmann ${ }^{\text {b }}$ \\ ${ }^{a}$ Instituto de Física, Universidade Federal do Rio Grande do Sul, \\ 91501-970 Porto Alegre, Brazil \\ ${ }^{\mathrm{b}}$ Institut für Computerphysik, Universität Stuttgart, \\ D-70569 Stuttgart, Germany
}

\begin{abstract}
We report surprisingly regular behaviors observed for a class 4 cellular automaton, the totalistic rule 20: starting from disordered initial configurations the automaton produces patterns which are periodic not only in time but also in space. This is the first evidence that different types of spatio-temporal order can emerge under specific conditions out of disorder in the same discrete rule based algorithm.
\end{abstract}

Cellular automata (CA) are discrete dynamical systems of many degrees of freedom that have been used successfully to describe a plethora of nonlinear phenomena ranging from pattern formation to chaos, have been applied for modelization in biology, geology, chemistry, sociology, etc and have even been proposed as minimal computer. In a seminal and remarkably influential work Wolfram [1,2] distinguished four classes of behavior for binary CA, i.e. those with a local degree of freedom $\sigma_{i}$ which is either $\sigma_{i}=1$ or $\sigma_{i}=0$. In class 1 all initial configurations evolve to a fixed point, when all cells are either one or zero. Automata of class 2 evolve to spatially static structures with eventually short-lived temporal oscillations. Class 3 automata produce selfsimilar patterns that can be characterized as discrete chaos. Finally, the most interesting class 4 contains all remaining automata which, according to Wolfram, display behaviors "more complex than chaos" being possibly capable of universal computation. The famous Game of Life is a CA of class 4 [5].

Wolfram has recently evoked class 4 automata as paradigms for the emergence of life[2]. Our findings support this picture in the sense that specific initial conditions in confined systems yield highly non-trivial spatio-temporal periodic patterns while the overall picture is dominated by long transients. In

* Corresponding author: jgallas@if.ufrgs.br 

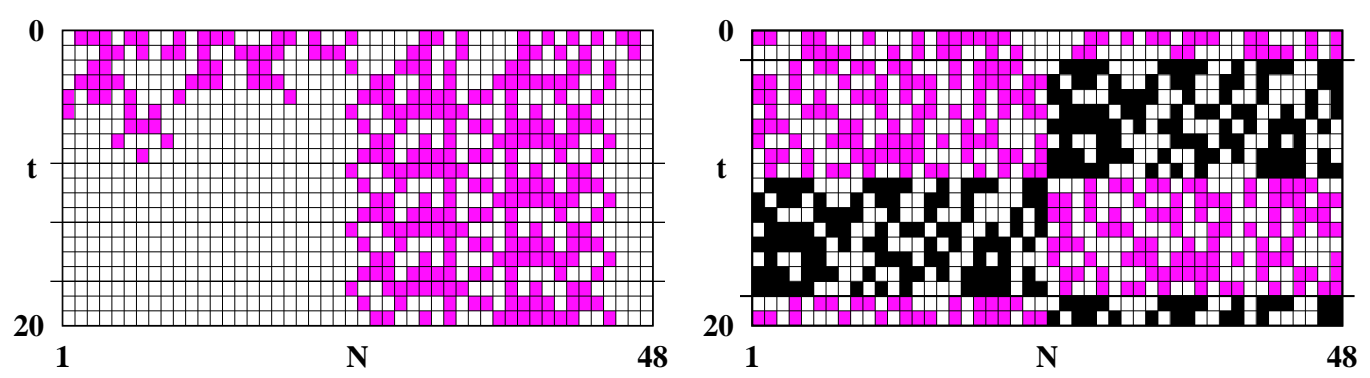

Fig. 1. Left: a typical period-4 persistent structure ("glider") emerging after a transient. Right: example of a remarkably regular space-filling pattern of period 16 . Two tonalities are used to represent " 1 " to better display inner symmetries.

fact, based on the behavior of the class 4 rule 20 automaton it has been argued [6] that in the thermodynamic limit, the limit of infinite lattice size, class 4 automata are just having extremely long transients but ending invariably in one of the three simpler classes.

The purpose of this short note is to illustrate graphically a number of very regular behaviors that we observed and are studying on class 4 automata. All results here are for rule 20 although similar behavior exists for other rules.

The remarkable new behavior observed is that starting from disordered initial configurations one finds patterns which are periodic not only in time but also in space. We refer to such behavior as spatio-temporal order. We believe this to be the first evidence that different types of spatio-temporal order can emerge under specific conditions out of disorder in the same discrete rule based algorithm. It also shows that although dependent on the initial condition, class 4 automata can yield highly regular attractors which have not been observed before, not even for the three simpler classes. This behavior is not to be confused with trivial static backgrounds familiar from, e.g., nearest-neighbor 1D rule 110. Interestingly, spatio-temporal order seems to predominate for smaller systems since preliminary data suggest that the probability of finding then may vanish in the thermodynamic limit, thereby not excluding the speculation about intrinsic transient behavior in class 4 automata [6].

Our cellular automaton is a linear chain of $N$ sites with the aforementioned binary degree of freedom $\sigma_{i}$ and periodic boundary conditions. The $\sigma_{i}$ values define the state of each site at a given time. For rule 20 the state of any given site $i$ at time $t+1$ depends on the state of the site at time $t$ as well as on the state of its nearest and next-nearest neighbors through the sum $\Sigma \equiv \sigma_{i-2}(t)+\sigma_{i-1}(t)+\sigma_{i}(t)+\sigma_{i+1}(t)+\sigma_{i+2}(t)$ and is synchronously updated as follows:

$$
\sigma_{i}(t+1)= \begin{cases}1 & \text { if } \Sigma=2 \text { or } 4 \\ 0 & \text { otherwise }\end{cases}
$$



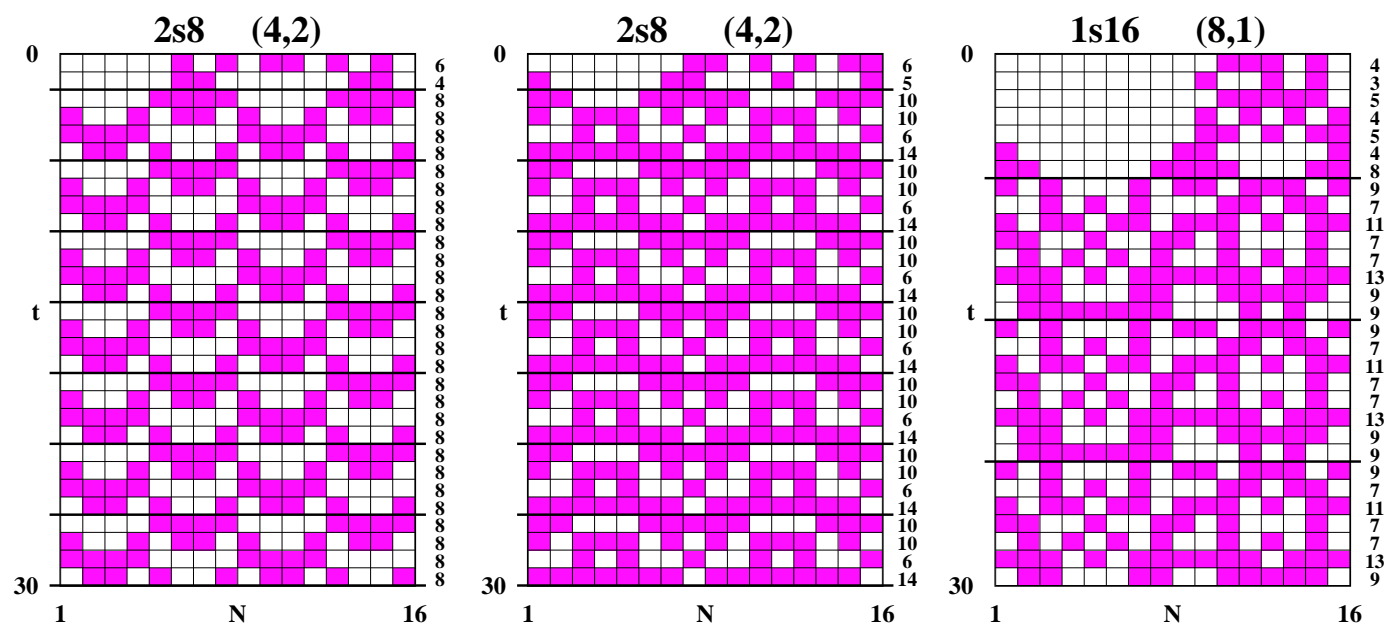

Fig. 2. Spatially primitive and doubling patterns obtained for $N=16$ sites. The rightmost figure shows a primitive pattern of spatial period 16 , denoted $1 s 16$, while the other two figures display spatial-doublings, denoted $2 s 8$, of primitive patterns of spatial period 8 . The notation " $(4,2)$ " indicates a pattern 4-periodic in time and 2-periodic in space. Vertical numbers on the right give the "magnetization", i.e. the total amount of "1" at each time step.

The system is started at $t=0$ from a random initial configuration. The leftmost portion of Fig. 1 shows a typical example of the non-trivial behaviors studied by Wolfram, a so-called glider $[1,2,3,4]$, a localized structure that propagates along the automaton. The rightmost portion of Fig. 1 shows an example of the new behaviors reported here: the spontaneous emergence of spatio-temporal order in the automaton, following a transient "equilibration" time. The great regularity seen in the rightmost pattern in Fig. 1 contrasts sharply with "more complex than chaos" expectations for class 4 .

Figure 2 shows some space-filling patterns obtained for a lattice with $N=16$ sites. The two leftmost patterns contain spatial-doublings of two different primitive patterns observed for $N=8$. Noteworthy is the fact that despite asymmetries in the initial conditions, spatial periodicity sets in. On the rightmost pattern, notice the relatively long lack of activity in half of the figure.

Figure 3 shows that it is possible to tile periodically the space when $N$ grows. We stress that initial conditions are always random. As the figure for $N=18$ shows, in some cases the transient may be several times bigger than the final temporal periodicity that sets in. Figure 4 illustrates several interesting things. It shows that spatial periodicity may be easily induced by "damaging" the automata in randomly chosen sites. The black cells indicate the time and position of the damage, the actual damage consisting in reversing the state of the site after having updated it using Eq. (1). Figure 4 also shows that damage may not only induce transitions among different patterns but is capable of inducing changes in the spatial periodicity of the system, in particular a frequently observed result is to see doublings of the spatial period. Figures 5 

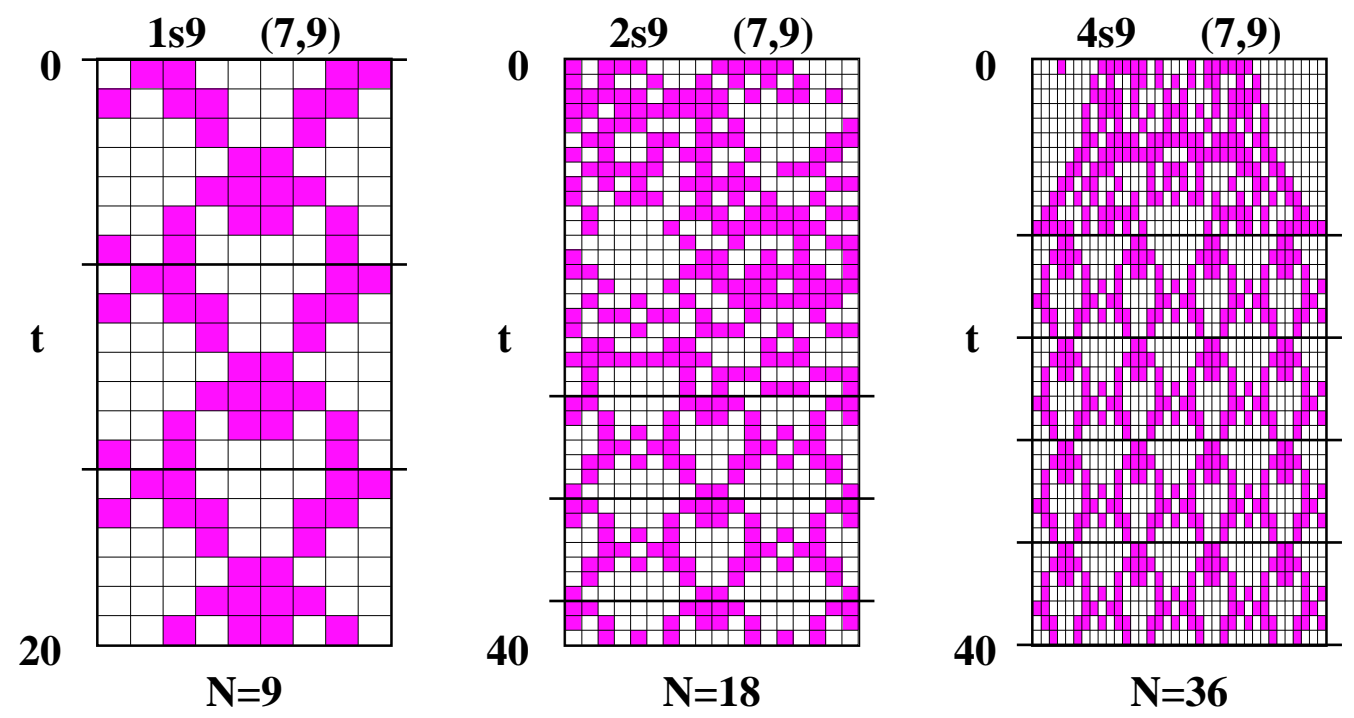

Fig. 3. Spatial doublings $\ell s 9$, where $\ell=1$ indicates a primitive spatial period, $\ell=2$ indicates a doubling, etc. Here temporal and spatial periodicities produce a "(7,9)-pattern" namely, a pattern which is 7-periodic in time and 9-periodic in space. Initial conditions are generated randomly.
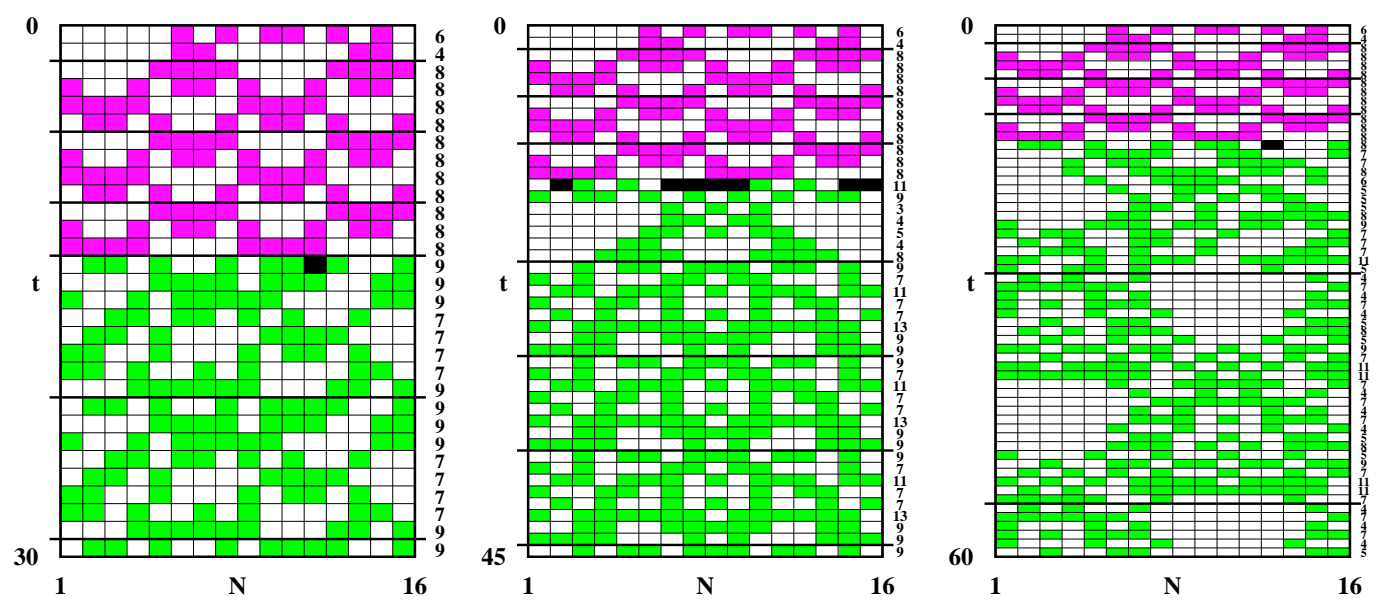

Fig. 4. Effect of low and high damage (in black) in the evolution of a given pattern.

and 6 are explained in their captions. We conclude recalling that small lattices and relatively short temporal evolutions like those reported here are precisely the most interesting regimes for applications [2,3,4]. A promising possibility seems to be exploiting class 4 automata as a tool to compress digital sound and images. Detailed results will be reported elsewhere.

JACG thanks Sonderforschungsbereich 404, Germany, and CNPq, Brazil, for financial support. HJH thanks the Max-Planck-Forschungspreis awarded to him. 


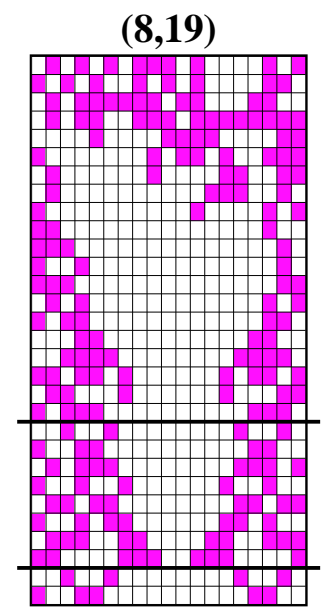

$\mathbf{N}=19$

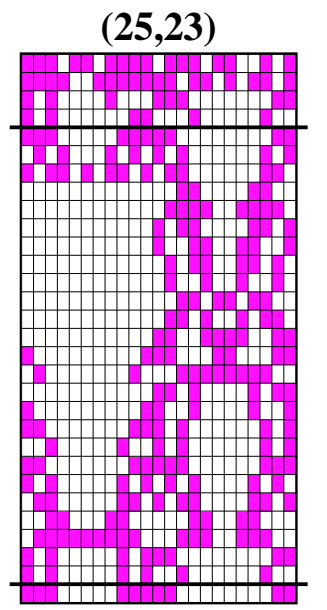

$\mathrm{N}=\mathbf{2 3}$
$(14,29)$

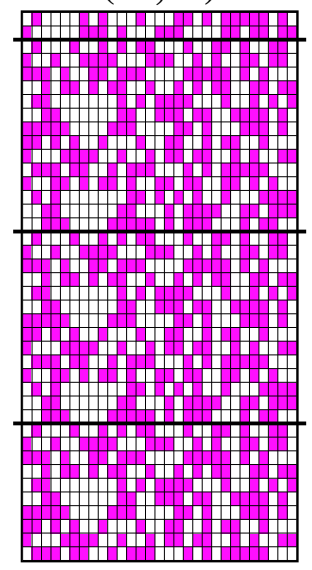

$\mathbf{N}=29$
$(30,31)$

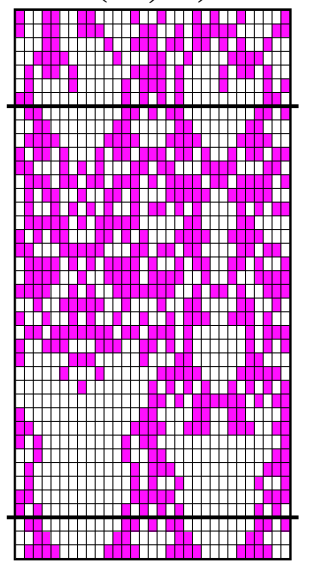

$\mathbf{N}=31$

Fig. 5. Primitive periods for odd lattice sizes, shown for 30 time steps. The structure seen for $N=19$ grows until it touches itself and contracts, pulsating periodically.
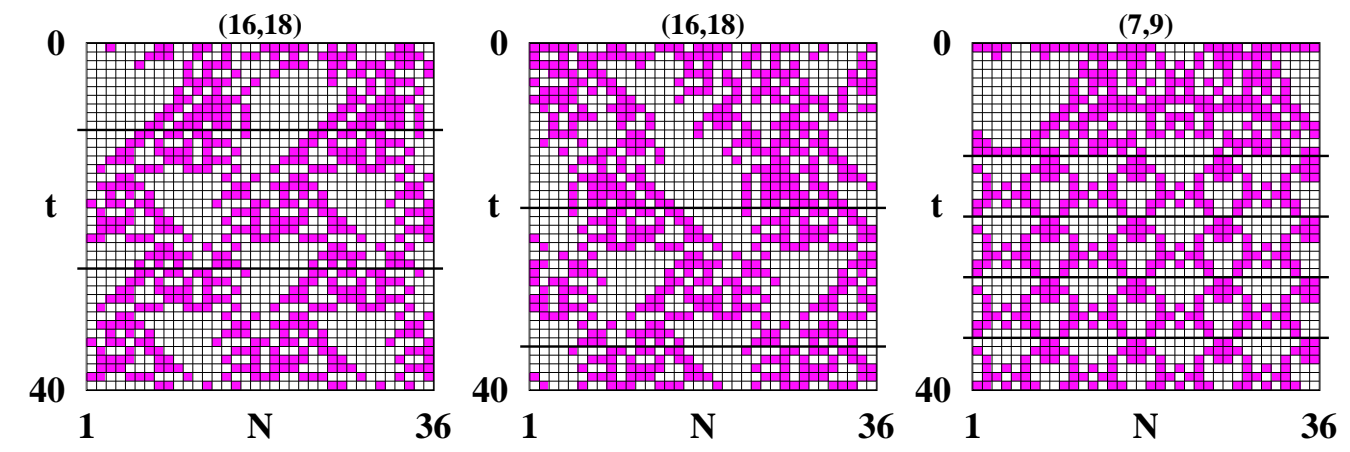

Fig. 6. Different transients, similar starting phases and strongly correlated spatial patterns. Compare the $(7,9)$ pattern here with the corresponding $4 s 9$ in Fig. 3. Note the common precursor "gun" emitting periodic gliders. The precursor here has quite distinct initial conditions. Another similar precursor may be seen in Fig. (4).

\section{References}

[1] S. Wolfram, Universality and complexity in CA, Physica D, 10 (1984) 1-35.

[2] S. Wolfram, A New Kind of Science, Wolfram Media Inc, Champaign, 2002.

[3] A. Ilachinski, Cellular Automata, World Scientific, Singapore, 2001.

[4] B. Chopard and M. Droz, Cellular automata modeling of physical systems, Cambrige University Press, Cambridge, 1998.

[5] H.V. McIntosh, Wolfram's class IV automata and a good life, Physica D, 45 (1990) 105-121.

[6] J.A.C. Gallas and H.J. Herrmann, Investigating an automaton of class 4, Int. J. Mod. Phys. C, 1 (1990) 181-191. This paper is available at: http://www.icp.uni-stuttgart.de/ jgallas/CA 